COMMUNICATIONS IN

ANALYSIS AND GEOMETRY

Volume 15, Number 1, 1-28, 2007

\title{
Admissible wavelets and inverse radon transform associated with the affine homogeneous Siegel domains of type II
}

\author{
JiAnXun He AND HePing LiU
}

\begin{abstract}
Let $D(\Omega, \Phi)$ be the affine homogeneous Siegel domain of type II, whose Silov boundary $N$ is a nilpotent Lie group of step two. In this article, we develop the theory of wavelet analysis on $N$. By selecting a set of mutual orthogonal wavelets we give a direct sum decomposition of $L^{2}(D(\Omega, \Phi))$, the first component $A_{0,0}^{0}$ of which is the Bergman space. Moreover, we study the Radon transform on $N$, and obtain an inversion formula $R^{-1}=(\pi)^{-2 d} L R L$ which is an extension of that by Strichartz [R. S. Strichartz, $L^{p}$ harmonic analysis and Radon transforms on the Heisenberg group, J. Funct. Anal. 96 (1991), 350-406.]. We devise a subspace of $L^{2}(N)$ on which the Radon transform is a bijection. Using wavelet inverse transform, we establish an inversion formula of the Radon transform in the weak sense.
\end{abstract}

\section{Introduction}

The wavelet transform is a very useful analysis tool in pure and applied mathematics. The research of this subject on Euclidean space has made considerable progress $[2,4]$ and the references therein. It is well known that one-dimensional wavelet analysis can be explained in terms of square integrable representations associated with the affine automorphism group of the upper half plane $[1,5,10,14]$. More precisely, the continuous (admissible) wavelets are closely related to square integrable representation of a non-unimodular group. For this we refer the reader to see $[6,8]$. In this viewpoint, various authors extended the theory of wavelet analysis on real line $\mathbb{R}$ to the tube domain $[17,20]$ and unbounded realization of the unit ball in $\mathbb{C}^{n}[11,21]$. The Radon transform has also received considerable attention in mathematical literature due to its wide applications to partial differential 
equations, X-ray technology, radio astronomy and so on. For the basic theory and further results of the Radona transform, we refer the reader to the book [15] by Helgason and the references therein. A combined use of the Radon transform and the wavelet transform can be called "wavelet Radon transform" [31] which has proved to be very useful both in pure mathematics and its applications. Recently, some authors deal with the inversion formula of Radon transforms by using inverse wavelet transforms. Holschneider [16] considered the classical Radon transform on the two-dimensional plane. His results were extended by Rubin $[26,27]$ to the $k$-dimensional Radon transform on $\mathbb{R}^{n}$ and totally geodesic Radon transform on the sphere and hyperbolic space. Strichartz [29] defined the Radon transform on the Heisenberg group $H_{n}$ and gave an inversion formula. Nessibi and Trimèche [23] obtained an inversion formula of the Radon transform on the Laguerre hypergroup $K=[0, \infty) \times \mathbb{R}$ by use of the generalized wavelet transform.

The function theory on Siegel domain $D(\Omega, \Phi)$ of type II has always exerted a strong attraction due to its important geometric background. Many classical results have been extended to this case. It is a well-known fact that the distinguished boundary or S̆ilov boundary $N$ of $D(\Omega, \Phi)$ is a nilpotent Lie group of step two. The theory of harmonic analysis and other problems on $N$ were considered in $[3,24,30]$. In this paper, we develop a theory of wavelet transform and Radon transform for the affine homogeneous Siegel domains of type II. As applications for these wavelets, we give a direct sum orthogonal decomposition for $L^{2}(D(\Omega, \Phi))$. Moreover, we obtain an inversion of the Radon transform by using the inverse wavelet transform. The harmonic analysis on a nilpotent Lie group developed by Ogden and Vági [24] plays an important role in this paper.

This article is organized as follows: in Section 2, we recall some basic facts relating to the group $N$, specifically including the Iwasawa subgroup $P$ of the automorphism group of affine homogeneous Siegel domain $D(\Omega, \Phi)$. In the third section, we define the unitary representations of $P$ on $L^{2}(N)$, and make a survey of harmonic analysis on $N$. We also give an irreducible decomposition of $L^{2}(N)$. Section 4 develops a theory of continuous wavelet analysis. In Section 5, we present the orthogonal sum decomposition for the function spaces $L^{2}(P)$ and $L^{2}(D(\Omega, \Phi))$ by using wavelet transforms. In the decomposition for $L^{2}(D(\Omega, \Phi))$, the first component is just the Bergman space. In Section 6, we investigate the Radon transform on $N$. Two function spaces $\mathscr{S}_{R}(N)$ and $L_{R}^{2}(N)$ are introduced on which the Radon transform $R$ is a bijection. The inversion formula for the Radon transform $R^{-1}=$ $(\pi)^{-2 n} L R L$ holds on $\mathscr{S}_{R}(N)$. In Section 7 , we make use of suitable wavelets to derive an inversion formula of the Radon transform on $L_{R}^{2}(N)$ in the weak 
sense. Finally, in Section 8 we state our results in a more explicit form in terms of Jordan algebra for the symmetric Siegel domain of type II, which is the most interested case.

\section{The affine homogeneous Siegel domains of type II}

We start with some notations and facts on Siegel domains of type II, especially the Iwasawa subgroup of the affine automorphism group of an affine homogeneous Siegel domain of type II. Further details can be found in [18, $22,25,30]$.

Let $U$ be the $m$-dimensional Euclidean space. A regular cone $\Omega$ in $U$ is a non-empty convex open cone which contains no entire straight line. Let $V$ be the $n$-dimensional complex vector space. $W$ denotes the complexification of $U$. A map $\Phi$ of $V \times V$ into $W$ is called an $\Omega$-positive Hermitian map if the following conditions are satisfied:

(i) $\Phi(\zeta, \eta)$ is $\mathbb{C}$-linear in $\zeta$.

(ii) $\overline{\Phi(\zeta, \eta)}=\Phi(\eta, \zeta)$.

(iii) $\Phi(\zeta, \zeta) \in \bar{\Omega}$.

(iv) $\Phi(\zeta, \zeta)=0$ only if $\zeta=0$.

Then, the Siegel domain of type II $D=D(\Omega, \Phi)$ determined by a regular cone $\Omega$ and an $\Omega$-positive Hermitian map $\Phi$ is defined by

$$
D=\{(z, \zeta) \in W \times V: \operatorname{Im} z-\Phi(\zeta, \zeta) \in \Omega\}
$$

The Šilov boundary $S$ of $\Omega$ is given by

$$
S=\{(z, \zeta) \in W \times V: \operatorname{Im} z-\Phi(\zeta, \zeta)=0\}
$$

Let $G_{a}(D)$ be the affine automorphism group of $D . G_{a}(D)$ can be decomposed into the semi-direct product $G_{a}(D)=N H$ of the subgroups $N$ and $H$. Here $N$ is a simply connected nilpotent Lie group of step two with the underlying manifold $U \times V$ and the multiplication

$$
(a, \alpha)(b, \beta)=(a+b+2 \operatorname{Im} \Phi(\alpha, \beta), \alpha+\beta), \quad(a, \alpha),(b, \beta) \in U \times V
$$

and $H=G L(W \times V) \bigcap G_{a}(D)$. $H$ consists of all pairs $(A, B)$ where $A$ is in the automorphism group $\operatorname{Aut}(\Omega)$ of $\Omega$, the subgroup of $G L(U)$ which leaves 
$\Omega$ invariant and $B \in G L(V)$ such that

$$
A \Phi(\zeta, \eta)=\Phi(B \zeta, B \eta), \quad \zeta, \eta \in V .
$$

The actions of $N$ and $H$ on $D($ and $S$ ) are separately given by

$$
(z, \zeta) \mapsto(a, \alpha)(z, \zeta)=(a+z+2 i \Phi(\zeta, \alpha)+i \Phi(\alpha, \alpha), \zeta+\alpha)
$$

and

$$
(z, \zeta) \mapsto(A, B)(z, \zeta)=(A z, B \zeta)
$$

We assume that $D$ is affine homogeneous, that is, $G_{a}(D)$ acts on $D$ transitively. We define a homomorphism $\mu$ of $H$ into $\operatorname{Aut}(\Omega)$ by $\mu(A, B)=A$. The affine homogeneity of $D$ is characterized by the transitivity of $\mu(H)$, i.e., $G_{a}(D)$ acts on $D$ transitively if and only if $\mu(H)$ acts on $\Omega$ transitively. Specifically, all symmetric Siegel domains are affine homogeneous, which will be treated more explicitly in Section 8. An example of affine homogeneous Siegel domain which is non-symmetric can be found in [22]. Let $G_{a}(D)^{0}$ be the identity component of $G_{a}(D)$. Then $G_{a}(D)^{0}$ also acts on $D$ transitively. Fix a point $e \in \Omega$. Let $K$ be the isotropy subgroup of $G_{a}(D)$ at the point $(i e, 0) \in D . H^{0}$ and $K^{0}$ are the identity components of $H$ and $K$, respectively. Then $K^{0} \subset H^{0}$ and $K^{0}$ is a maximal compact subgroup of $G_{a}(D)^{0}$ (and $H^{0}$ ). Therefore $H^{0}=T_{1} K^{0}$ (semi-direct product) where $T_{1}$ is a maximal $\mathbb{R}$-triangular subgroup of $H$. The kernel of $\mu$ is in $K$. $\mu(H)$ acts on $\Omega$ transitively and $\mu(K)$ is the isotropy subgroup of $\mu(H)$ at the point $e \in \Omega$. Therefore $T=\mu\left(T_{1}\right)$ is a maximal $\mathbb{R}$-triangular subgroup of $\operatorname{Aut}(\Omega)$ and $\mu$ gives an isomorphism of $T_{1}$ and $T$. The elements of $T_{1}$ can be written in the form $(t, B(t))$ where $t \in T$ and $B(t) \in G L(V)$ are uniquely determined by $t$ such that

$$
t \Phi(\zeta, \eta)=\Phi(B(t) \zeta, B(t) \eta), \quad \zeta, \eta \in V .
$$

Let $P=N T_{1}$. $P$ is called the Iwasawa subgroup of $G_{a}(D)$. The action of $P$ on $D$ is given by

$$
(z, \zeta) \mapsto(a, \alpha, t)(z, \zeta)=(a+t z+2 i \Phi(B(t) \zeta, \alpha)+i \Phi(\alpha, \alpha), B(t) \zeta+\alpha) .
$$

The multiplication of $P$ is given by

$$
(a, \alpha, t)(b, \beta, s)=(a+t b+2 \operatorname{Im} \Phi(\alpha, B(t) \beta), \alpha+B(t) \beta, t s) .
$$


Let Det denote the determinant of a linear transformation (or a matrix). From (2.7) it is not difficult to get

$$
\operatorname{Det}(t)^{n}=|\operatorname{Det} B(t)|^{2 m} \text {. }
$$

Then, the left Haar measure of $P$ turns out to be $\operatorname{Det}(t)^{-\frac{m+n}{m}} d a d \alpha d m_{l}(t)$ where $d a d \alpha$ denotes the Haar measure of $N$ which coincides with the Lebesgue measure of $U \times V$ and $d m_{l}(t)$ is the left Haar measure of $T$. Obviously $P$ acts on $D$ simply transitively. We can identify $P$ with $D$ by identification of $(a, \alpha, t)$ and $(a+i(t e+\Phi(\alpha, \alpha)), \alpha)$. We can also identify $N$ with $S$ by identification of $(a, \alpha)$ and $(a+i \Phi(\alpha, \alpha), \alpha)$.

\section{The decomposition of $L^{2}(N)$}

$P$ has a natural unitary representation $\pi$ on $L^{2}(N)$ (or $L^{2}(S)$ instead) which is defined by

$$
\begin{aligned}
\left(\pi_{(a, \alpha, t)} f\right)(x, \zeta)= & \operatorname{Det}(t)^{-((m+n) / 2 m)} f\left((a, \alpha, t)^{-1}(x, \zeta)\right) \\
= & \operatorname{Det}(t)^{-((m+n) / 2 m)} f\left(t^{-1}(x-a\right. \\
& \left.-2 \operatorname{Im} \Phi(\alpha, \zeta), B(t)^{-1}(\zeta-\alpha)\right)
\end{aligned}
$$

This section is devoted to decompose $L^{2}(N)$ into the direct sum of the irreducible invariant closed subspaces under $\pi$. We shall make use of the harmonic analysis on the nilpotent Lie group $N$ which is due to Ogden and Vági [24].

Let $U^{\prime}$ denote the (real) dual of $U$. The adjoint action of $T$ on $U^{\prime}$ is given by

$$
\lambda \mapsto t^{*-1} \lambda, \quad t \in T, \quad \lambda \in U^{\prime},
$$

where $t^{*}$ is the adjoint of $t$. Suppose $\Lambda_{\epsilon}$ is a $T$-orbit of $U^{\prime}$ under the adjoint action. Because $T$ is a connected $\mathbb{R}$-triangular group, $\Lambda_{\epsilon}$ has positive Lebesgue measure if and only if the adjoint action of $T$ on $\Lambda_{\epsilon}$ is simple. Let $\left\{\Lambda_{\epsilon}: \epsilon \in E\right\}$ is the set of all simple $T$-orbits of $U^{\prime}$ under the adjoint action. Then $\Lambda=\cup_{\epsilon \in E} \Lambda_{\epsilon}$ has total Lebesgue measure in $U^{\prime}$. The parametric representation of $E$ for symmetric case is given in Section 8. It is usually obvious for every concrete case.

For $\lambda \in U^{\prime}$, we set

$$
H_{\lambda}(\zeta, \eta)=4\langle\lambda, \Phi(\zeta, \eta)\rangle
$$


and

$$
B_{\lambda}(\zeta, \eta)=\operatorname{Im}\left(H_{\lambda}(\zeta, \eta)\right)
$$

The Hermitian form $H_{\lambda}$ is non-degenerate for $\lambda \in U^{\prime}$ almost everywhere. Note that

$$
H_{t^{*} \lambda}(\zeta, \eta)=H_{\lambda}(B(t) \zeta, B(t) \eta)
$$

It is easy to see that $H_{\lambda}$ is non-degenerate if $\lambda \in \Lambda$. Fix a complex basis $\beta_{1}, \ldots, \beta_{n}$ for $V$ which is compatible with the chosen Lebesgue measure $d \zeta$ of $V$. Let $M_{\lambda}(\beta)$ be the matrix defined by $\left\langle\lambda, \Phi\left(\beta_{i}, \beta_{j}\right)\right\rangle$. For $\lambda \in \Lambda$, we define

$$
\rho(\lambda)=4^{n}\left|\operatorname{Det} M_{\lambda}(\beta)\right|
$$

Then $\rho(\lambda)$ is a positive continuous function on $\Lambda$ independent of the compatible basis chosen. $\rho(\lambda) d \lambda$ is essentially the Plancherel measure for $N$. Fix a point $\lambda_{\epsilon} \in \Lambda_{\epsilon}$ for each simple $T$-orbit $\Lambda_{\epsilon}$. Select a complex basis $\beta_{1}\left(\lambda_{\epsilon}\right), \ldots, \beta_{n}\left(\lambda_{\epsilon}\right)$ for $V$ such that

$$
H_{\lambda_{\epsilon}}\left(\beta_{i}\left(\lambda_{\epsilon}\right), \beta_{j}\left(\lambda_{\epsilon}\right)\right)=\sigma_{i} \delta_{i j}
$$

where $\sigma_{i}= \pm 1$ and $\delta_{i j}$ is the Kronecker symbol. Suppose $\lambda=t^{*} \lambda_{\epsilon} \in \Lambda_{\epsilon}$. Set $\beta_{j}(\lambda)=B(t)^{-1} \beta_{j}\left(\lambda_{\epsilon}\right), j=1, \ldots, n$. Then $\beta(\lambda)$ is a complex basis of $V$ such that

$$
H_{\lambda}\left(\beta_{i}(\lambda), \beta_{j}(\lambda)\right)=\sigma_{i} \delta_{i j}
$$

Let $V^{\mathbb{R}}$ be the underlying real space of $V$ and $J$ be the original complex structure of $V$. Then $\beta_{1}(\lambda), \ldots, \beta_{n}(\lambda), J \beta_{1}(\lambda), \ldots, J \beta_{n}(\lambda)$ is a basis of $V^{\mathbb{R}}$. Let $J_{\lambda}: V^{\mathbb{R}} \mapsto V^{\mathbb{R}}$ be defined in the $\beta(\lambda)$-basis by the $2 n \times 2 n$ matrix

$$
J_{\lambda}=\left(\begin{array}{cc}
0 & -\sigma \\
\sigma & 0
\end{array}\right)
$$

with $\sigma=\operatorname{diag}\left(\sigma_{1}, \sigma_{2}, \ldots, \sigma_{n}\right)$. Then $J_{\lambda}$ is a complex structure which commutes with $J$. We denote $V^{\mathbb{R}}$ equipped with the complex structure $J_{\lambda}$ by $V_{\lambda}$. Let $E_{\lambda}$ be the real span of $\beta_{1}(\lambda), \ldots, \beta_{n}(\lambda)$, then $V_{\lambda}=E_{\lambda} \oplus J_{\lambda} E_{\lambda}$. Suppose $\zeta=\xi+J_{\lambda} \theta$, where $\xi=\sum_{j=1}^{n} \xi_{j} \beta_{j}(\lambda) \in E_{\lambda}, \theta=\sum_{j=1}^{n} \theta_{j} \beta_{j}(\lambda) \in E_{\lambda}$. Set $\bar{\zeta}=$ 
$\xi-J_{\lambda} \theta, \zeta_{j}=\xi_{j}+i \theta_{j}$. We define

$$
\zeta \cdot \eta=B_{\lambda}\left(J_{\lambda} \zeta, \bar{\eta}\right)+i B_{\lambda}(\zeta, \bar{\eta})
$$

and

$$
|\zeta|^{2}=\zeta \cdot \bar{\zeta}
$$

Then we have $\zeta \cdot \eta=\sum_{j=1}^{n} \zeta_{j} \eta_{j}$ and $|\zeta|^{2}=\sum_{j=1}^{n}\left|\zeta_{j}\right|^{2}$.

If $\tau \in E_{\lambda}$, then $\tau=\sum_{j=1}^{n} \tau_{j} \beta_{j}(\lambda)$. We also write $\tau=\left(\tau_{1}, \tau_{2}, \ldots, \tau_{n}\right)$ to denote the coordinates of $\tau$ under the basis $\beta_{1}(\lambda), \beta_{2}(\lambda), \ldots, \beta_{n}(\lambda)$. Now let $\zeta=\xi+J_{\lambda} \theta$, then $\xi=\sum_{j=1}^{n} \xi_{j} \beta_{j}(\lambda)$ and $\theta=\sum_{j=1}^{n} \theta_{j} \beta_{j}(\lambda)$. Letting $g(\zeta) \in$ $L^{1}(V)$, we have

$$
\int_{V} g(\zeta) \rho(\lambda) d \zeta=\int_{E_{\lambda} \times E_{\lambda}} g\left(\xi+J_{\lambda} \theta\right) \vartheta_{\lambda}(d \xi) \vartheta_{\lambda}(d \theta)
$$

where $\vartheta_{\lambda}(d \xi)=d \xi_{1} d \xi_{2} \cdots d \xi_{n}$. Let $\psi_{m}(t)$ be the normalized Hermite function defined by

$$
\psi_{m}(t)=\left(2^{1 / 4} 2^{-m / 2}(m !)^{-1 / 2}\right) h_{m}\left((2 \pi)^{(1 / 2)} t\right) \exp \left(-\pi t^{2}\right),
$$

where $h_{m}$ denotes the classical Hermite polynomial. Let $\nu=\left(\nu_{1}, \nu_{2}, \ldots, \nu_{n}\right)$ be a multi-index, the higher dimensional Hermite functions $\Phi_{\nu, \lambda}$ is defined by tensor products, i.e.,

$$
\Phi_{\nu, \lambda}(\tau)=\prod_{j=1}^{n} \psi_{\nu_{j}}\left(\tau_{j}\right) .
$$

Obviously, $\left\{\Phi_{\nu, \lambda}(\tau): \nu \in \mathbb{Z}_{+}^{n}\right\}$ forms an orthonormal basis for Hilbert space $\mathscr{H}_{\lambda}=L^{2}\left(E_{\lambda}, \vartheta_{\lambda}(d \tau)\right)$. The Schrödinger representation of $N$ on $\mathscr{H}_{\lambda}$ is defined by

$$
\left(\pi^{\lambda}(x, \zeta) \phi\right)(\tau)=\exp (-2 \pi i\langle\lambda, x\rangle) \exp (\pi i \xi \cdot \eta) \exp (-2 \pi i \eta \cdot \tau) \phi(\tau-\xi),
$$

where $\zeta=\xi+J_{\lambda} \eta, \tau \in E_{\lambda}, \phi \in \mathscr{H}_{\lambda}$. Thus $\pi^{\lambda}(x, \zeta)$ is an irreducible unitary representation of the group $N$ on $\mathscr{H}_{\lambda}$. The Fourier transform of a function $f \in L^{1}(N)$ is an operator valued function defined by

$$
\widehat{f}(\lambda)=\int_{N} f(x, \zeta) \pi^{\lambda}(x, \zeta) d x d \zeta
$$


We have the Plancherel formula

$$
\|f\|_{L^{2}(N)}=\left(\int_{\Lambda}\|\widehat{f}(\lambda)\|_{\mathrm{HS}}^{2} \rho(\lambda) d \lambda\right)^{(1 / 2)}, \quad f \in L^{1}(N) \cap L^{2}(N),
$$

where $\|\cdot\|_{\text {HS }}$ denotes the Hilbert-Schmidt norm of an operator. The Plancherel formula is equivalent to

$$
\langle f, g\rangle_{L^{2}(N)}=\int_{\Lambda} \operatorname{tr}\left(\widehat{g}(\lambda)^{*} \widehat{f}(\lambda)\right) \rho(\lambda) d \lambda, \quad f, g \in L^{1}(N) \cap L^{2}(N),
$$

which allows us to extend the Fourier transform to the tempered distributions on $N$ by duality.

Let $f * g$ be the convolution of $f$ and $g$ defined by

$$
f * g(x, \zeta)=\int_{N} f(y, \eta) g\left((y, \eta)^{-1}(x, \zeta)\right) d y d \eta
$$

Set

$$
\widetilde{f}(x, \zeta)=\overline{f\left((x, \zeta)^{-1}\right)}=\overline{f(-x,-\zeta)} .
$$

It is easy to see that

$$
\widehat{f * g}(\lambda)=\widehat{f}(\lambda) \widehat{g}(\lambda)
$$

and

$$
\widehat{\widetilde{f}}(\lambda)=\widehat{f}(\lambda)^{*}
$$

Suppose $t \in T$, we have the following identities:

$$
\begin{aligned}
J_{t^{*} \lambda} & =B(t)^{-1} J_{\lambda} B(t), \\
B_{t^{*} \lambda}(\zeta, \eta) & =B_{\lambda}(B(t) \zeta, B(t) \eta), \\
\rho\left(t^{*} \lambda\right) & =|\operatorname{Det} B(t)|^{2} \rho(\lambda) .
\end{aligned}
$$

The map

$$
F(\zeta) \mapsto G(\zeta)=F(B(t) \zeta)
$$

gives the isometrically isomorphism from $\mathscr{H}_{\lambda}$ onto $\mathscr{H}_{t^{*} \lambda}$. We can identify $\mathscr{H}_{\lambda}$ with $\mathscr{H}_{t^{*} \lambda}$. This means that we identify $\Phi_{\nu, \lambda}$ with $\Phi_{\nu, t^{*} \lambda}$. Then we have

$$
\pi^{t^{*} \lambda}(x, \zeta)=\pi^{\lambda}(t x, B(t) \zeta)
$$


Let

$$
f_{t}(x, \zeta)=\operatorname{Det}(t)^{-((m+n) / m)} f\left(t^{-1} x, B(t)^{-1} \zeta\right)
$$

Then

$$
\widehat{f_{t}}(\lambda)=\widehat{f}\left(t^{*} \lambda\right)
$$

We define the operator $P_{\nu}^{\epsilon}$ on $L^{2}(N)$ in terms of the Fourier transform by

$$
\widehat{P_{\nu}^{\epsilon} f}(\lambda)= \begin{cases}\widehat{f}(\lambda) P_{\nu}, & \text { if } \lambda \in \Lambda_{\epsilon}, \\ 0, & \text { if } \lambda \notin \Lambda_{\epsilon},\end{cases}
$$

where $P_{\nu}$ denotes the orthogonal projection from $\mathscr{H}_{\lambda}$ to the one dimensional subspace $\mathscr{H}_{\lambda, \nu}$ spanned by $\Phi_{\nu, \lambda}$. It is clear that $P_{\nu}^{\epsilon}$ is an orthogonal projection. Let $H_{\nu}^{\epsilon}$ denote the range of $P_{\nu}^{\epsilon}$, i.e.,

$$
H_{\nu}^{\epsilon}=\left\{f \in L^{2}(N): \quad \widehat{f}(\lambda)=\widehat{f}(\lambda) P_{\nu} \text { and } \widehat{f}(\lambda)=0 \text { if } \lambda \notin \Lambda_{\epsilon}\right\}
$$

Then we have the following theorem.

Theorem 3.1. Each $H_{\nu}^{\epsilon}$ is an irreducible invariant closed subspace of $L^{2}(N)$ under the unitary representation $\pi$ of $P$ defined by (3.1), and we have the direct sum decomposition

$$
L^{2}(N)=\bigoplus_{\epsilon \in E, \nu \in \mathbb{Z}_{+}^{n}} H_{\nu}^{\epsilon}
$$

Proof. It is clear that the $H_{\nu}^{\epsilon}$ 's are mutually orthogonal closed subspaces of $L^{2}(N)$, and $L^{2}(N)$ is the direct sum of $H_{\nu}^{\epsilon}$ 's. We prove that $H_{\nu}^{\epsilon}$ is invariant and irreducible. By (3.15), we have

$$
(\pi(x, \zeta, t) \widehat{f})(\lambda)=\operatorname{Det}(t)^{\frac{m+n}{2 m}} \pi^{\lambda}(x, \zeta) \widehat{f}\left(t^{*} \lambda\right)
$$

Therefore $H_{\nu}^{\epsilon}$ is invariant under the unitary representation $\pi$. Let $A$ be a non-zero invariant closed subspace of $H_{\nu}^{\epsilon}$ under $\pi$ and $A^{\perp}$ the orthogonal complement of $A$ in $H_{\nu}^{\epsilon}$. Take a function $g \in A$, not identically zero. Suppose 
$f \in A^{\perp}$, then

$$
\langle f, \pi(x, \zeta, t) g\rangle_{L^{2}(N)}=0 .
$$

Since

$$
\langle f, \pi(x, \zeta, t) g\rangle_{L^{2}(N)}=\operatorname{Det}(t)^{((m+n) / 2 m)} f * \widetilde{g}_{t}(x, \zeta),
$$

by (3.12), (3.13) and (3.15),

$$
\int_{N}\langle f, \pi(x, \zeta, t) g\rangle_{L^{2}(N)} \pi^{\lambda}(x, \zeta) d x d \zeta=\operatorname{Det}(t)^{((m+n) / 2 m)} \widehat{f}(\lambda) \widehat{g}\left(t^{*} \lambda\right)^{*}
$$

Thus for any $t \in T$, we have

$$
\widehat{f}(\lambda) \widehat{g}\left(t^{*} \lambda\right)^{*}=0, \quad \text { a.e. } \lambda \in \Lambda_{\epsilon} .
$$

Suppose $\widehat{f}(\lambda) \neq 0$ for $\lambda \in \Gamma_{1}$, where $\Gamma_{1} \subset \Lambda_{\epsilon}$ is a set of positive measure. Since $g$ is not identically zero, there is a positive measure set $\Gamma_{2} \subset \Lambda_{\epsilon}$ such that $\widehat{g}(\lambda) \neq 0$ for $\lambda \in \Gamma_{2}$. Let $\Gamma_{1}^{0}$ and $\Gamma_{2}^{0}$ consist of points of density of $\Gamma_{1}$ and $\Gamma_{2}$, respectively. Because the adjoint action of $T$ on $\Lambda_{\epsilon}$ is transitive, there is $t_{0} \in T$ such that $\Gamma=\Gamma_{1}^{0} \cap\left(t_{0}^{*-1} \Gamma_{2}^{0}\right)$ has positive measure. Note that $\widehat{f}(\lambda)=\widehat{f}(\lambda) P_{\nu}, \widehat{g}\left(t^{*} \lambda\right)^{*}=P_{\nu} \widehat{g}\left(t^{*} \lambda\right)^{*}$. This implies that $\widehat{f}(\lambda) \widehat{g}\left(t_{0}^{*} \lambda\right)^{*} \neq 0$ for $\lambda \in \Gamma$, which contradicts (3.20). So we have $\widehat{f}(\lambda)=0$, a.e. $\lambda \in \Lambda_{\epsilon}$. Therefore $f$ is identically zero. This proves that $H_{\nu}^{\epsilon}$ is irreducible.

At the end of this section, we point out the following facts: the dual cone $\Omega^{*}$ of $\Omega$ is a simple $T$-orbit of $U^{\prime}$ under the adjoint action. $P_{0}$ is the orthogonal projection to the vacuum state. If $\Lambda_{0}=\Omega^{*}$, then $H_{0}^{0}$ is exactly the Hardy space $H^{2}(N)$. All subspaces $H_{\nu}^{\epsilon}$ can be explained in terms of the tangential Cauchy-Riemann operators (creation/annihilation operators) [24].

\section{Admissible wavelets and wavelet transforms}

Given $\nu \in \mathbb{Z}_{+}^{n}, \epsilon \in E$. The restriction of $\pi$ on $H_{\nu}^{\epsilon}$ is square integrable in the sense that there exists a function $\phi(\neq 0)$ in $H_{\nu}^{\epsilon}$ such that

$C_{\phi}=\frac{1}{\|\phi\|_{L^{2}(N)}^{2}} \int_{P}\left|\langle\phi, \pi(x, \zeta, t) \phi\rangle_{L^{2}(N)}\right|^{2} \operatorname{Det}(t)^{-((m+n) / m)} d x d \zeta d m_{l}(t)<\infty$.

(4.1) is called the admissibility condition, and $\phi$ is called an admissible wavelet if $\phi$ satisfies (4.1). Now we are going to give the characterization of the admissibility condition in terms of the Fourier transform. 
We denote by $A W_{\nu}^{\epsilon}$ the set of all admissible wavelets in $H_{\nu}^{\epsilon}$, i.e.,

$$
A W_{\nu}^{\epsilon}=\left\{\phi \in H_{\nu}^{\epsilon}: \phi \text { satisfies(4.1) }\right\}
$$

Then we have the following theorem.

Theorem 4.1. Suppose $\phi(\neq 0)$ in $H_{\nu}^{\epsilon}$. Then $\phi \in A W_{\nu}^{\epsilon}$ if and only if

$$
C_{\phi}=\int_{T}\left\|\widehat{\phi}\left(t^{*} \lambda_{\epsilon}\right)\right\|_{\mathrm{HS}}^{2} d m_{l}(t)<\infty .
$$

Proof. Suppose $\phi \in H_{\nu}^{\epsilon}$. Using (3.19) and the Plancherel formula, we get

$$
\begin{aligned}
\int_{P} & \left|\langle\phi, \pi(x, \zeta, t) \phi\rangle_{L^{2}(N)}\right|^{2} \operatorname{Det}(t)^{-((m+n) / m)} d x d \zeta d m_{l}(t) \\
& =\int_{T}\left(\int_{\Lambda}\left\|\widehat{\phi}(\lambda) \widehat{\phi}\left(t^{*} \lambda\right)^{*}\right\|_{\mathrm{HS}}^{2} \rho(\lambda) d \lambda\right) d m_{l}(t) \\
& =\int_{T}\left(\int_{\Lambda} \operatorname{tr}\left(\widehat{\phi}(\lambda)^{*} \widehat{\phi}(\lambda) \widehat{\phi}\left(t^{*} \lambda\right)^{*} \widehat{\phi}\left(t^{*} \lambda\right)\right) \rho(\lambda) d \lambda\right) d m_{l}(t) .
\end{aligned}
$$

Note that $\widehat{\phi}(\lambda)^{*} \widehat{\phi}(\lambda)=h(\lambda) P_{\nu}$, where $h(\lambda)=\|\widehat{\phi}(\lambda)\|_{\mathrm{HS}}^{2}$, and $h(\lambda)=0$ if $\lambda \notin$ $\lambda_{\epsilon}$. Hence

$$
\begin{aligned}
\int_{P} & \left|\langle\phi, \pi(x, \zeta, t) \phi\rangle_{L^{2}(N)}\right|^{2} \operatorname{Det}(t)^{-((m+n) / m)} d x d \zeta d m_{l}(t) \\
& =\int_{T}\left(\int_{\Lambda} h(\lambda) h\left(t^{*} \lambda\right) \rho(\lambda) d \lambda\right) d m_{l}(t) \\
& =\int_{\Lambda}\left(\int_{T} h\left(t^{*} \lambda\right) d m_{l}(t)\right) h(\lambda) \rho(\lambda) d \lambda \\
& =\left(\int_{T}\left\|\widehat{\phi}\left(t^{*} \lambda_{\epsilon}\right)\right\|_{\mathrm{HS}}^{2} d m_{l}(t)\right)\left(\int_{\Lambda}\|\widehat{\phi}(\lambda)\|_{\mathrm{HS}}^{2} \rho(\lambda) d \lambda\right) \\
& =\left(\int_{T}\left\|\widehat{\phi}\left(t^{*} \lambda_{\epsilon}\right)\right\|_{\mathrm{HS}}^{2} d m_{l}(t)\right)\|\phi\|_{L^{2}(N)}^{2},
\end{aligned}
$$

where in the third equality we have used the left invariance of $d m_{l}(t)$.

Let $\phi \in A W_{\nu}^{\epsilon}, f \in H_{\nu}^{\epsilon}$. We define the wavelet transform of $f$ with respect to $\phi$ by

$$
W_{\phi} f(x, \zeta, t)=\langle f, \pi(x, \zeta, t) \phi\rangle_{L^{2}(N)}
$$


Let $\phi, \psi \in A W_{\nu}^{\epsilon}$. We define the "inner product" of $\phi$ and $\psi$ on $A W_{\nu}^{\epsilon}$ by

$$
\langle\phi, \psi\rangle_{A W}=\int_{T} \operatorname{tr}\left(\widehat{\psi}\left(t^{*} \lambda_{\epsilon}\right)^{*} \phi\left(t^{*} \lambda_{\epsilon}\right)\right) d m_{l}(t) .
$$

From the theory of square-integrable representation of non-unimodular groups, which is due to Duflo and Moore [6], we have the following consequences of Theorem 4.1, which can also be proved directly.

Theorem 4.2. Let $\phi, \psi \in A W_{\nu}^{\epsilon}, f, g \in H_{\nu}^{\epsilon}$. Then we have

$$
\left\langle W_{\phi} f, W_{\psi} g\right\rangle_{L^{2}(P)}=\langle\psi, \phi\rangle_{A W}\langle f, g\rangle_{L^{2}(N)}
$$

In particular,

$$
\left\|W_{\phi} f\right\|_{L^{2}(P)}=C_{\phi}^{(1 / 2)}\|f\|_{L^{2}(N)} .
$$

Let $\mathscr{S}(N)$ denote the Schwartz space on $N$, then we have the following reproducing formula.

Theorem 4.3. Let $\phi \in A W_{\nu}^{\epsilon}, f \in H_{\nu}^{\epsilon}$. Then we have the following reproducing formula in the weak sense:

$$
\begin{aligned}
f(x, \zeta)= & C_{\phi}^{-1} \int_{P} W_{\phi} f(a, \alpha, t)(\pi(a, \alpha, t) \phi)(x, \zeta) \\
& \operatorname{Det}(t)^{-((m+n) / m)} d a d \alpha d m_{l}(t) .
\end{aligned}
$$

Specially, if $\quad \phi \in A W_{\nu}^{\epsilon} \cap \mathscr{S}(N), f \in H_{\nu}^{\epsilon} \cap \mathscr{S}(N)$, then the above formula (4.8) holds for all $(x, \zeta) \in N$.

\section{The orthogonal decomposition of $L^{2}(D(\Omega, \Phi))$}

Let $\left\{\psi_{\delta}: \delta \in \Delta\right\}$ be an orthonormal basis of $L^{2}\left(T, d m_{l}(t)\right)$. We define the functions $\phi_{\mu, \nu, \delta}^{\epsilon}$ in terms of the Fourier transform by

$$
\widehat{\phi}_{\mu, \nu, \delta}^{\epsilon}(\lambda)= \begin{cases}\psi_{\delta}(t) P_{\mu, \nu}, & \text { if } \lambda=t^{*} \lambda_{\epsilon} \in \Lambda_{\epsilon}, \\ 0, & \text { if } \lambda \notin \Lambda_{\epsilon},\end{cases}
$$

where $P_{\mu, \nu}$ is the partial isometric operator on $\mathscr{H}_{\lambda}$ defined by

$$
P_{\mu, \nu} M=\left\langle M, \Phi_{\mu, \lambda}\right\rangle_{\mathscr{H}_{\lambda}} \Phi_{\nu, \lambda}, \quad M \in \mathscr{H}_{\lambda} .
$$


It is easy to see that $\phi_{\mu, \nu, \delta}^{\epsilon} \in A W_{\mu}^{\epsilon}$ and $\left\{\phi_{\mu, \nu, \delta}^{\epsilon}: \epsilon \in E, \mu, \nu \in \mathbb{Z}_{+}^{n}, \delta \in \Delta\right\}$ is an orthonormal and complete set with respect to $\langle,\rangle_{A W}$. Set

$$
A_{\mu, \nu, \delta}^{\epsilon}=\left\{W_{\phi_{\mu, \nu, \delta}^{\epsilon}} f: f \in H_{\mu}^{\epsilon}\right\} .
$$

By Theorem 4.2, $A_{\mu, \nu, \delta}^{\epsilon} \subset L^{2}(P)$. We note that

$$
A_{\mu, \nu, \delta}^{\epsilon}=A_{0, \nu, \delta}^{\epsilon}, \quad \mu \in \mathbb{Z}_{+}^{n},
$$

because the restrictions of $\pi$ on $H_{\mu}^{\epsilon}$ are equivalent for different $\mu$. We write $A_{\nu, \delta}^{\epsilon}$ instead of $A_{\mu, \nu, \delta}^{\epsilon}$.

\section{Theorem 5.1.}

$$
L^{2}(P)=\bigoplus_{\epsilon \in E, \nu \in \mathbb{Z}_{+}^{n}, \delta \in \Delta} A_{\nu, \delta}^{\epsilon}
$$

Proof. It is easy to see that all subspaces $A_{\nu, \delta}^{\epsilon}$ 's are mutually orthogonal. Let $F(x, \zeta, t) \in L^{2}(P)$. Set $F_{t}(x, \zeta)=F(x, \zeta, t)$. For almost everywhere $t \in T$, $F_{t}(x, \zeta) \in L^{2}(N)$. By the Plancherel formula, we obtain

$$
\begin{aligned}
\|F\|_{L^{2}(P)}^{2}= & \int_{T}\left(\int_{N}\left|F_{t}(x, \zeta)\right|^{2} d x d \zeta\right) \operatorname{Det}(t)^{-((m+n) / m)} d m_{l}(t) \\
= & \int_{T}\left(\int_{\Lambda}\left\|\widehat{F}_{t}(\lambda)\right\|_{\mathrm{HS}}^{2} \rho(\lambda) d \lambda\right) \operatorname{Det}(t)^{-((m+n) / m)} d m_{l}(t) \\
= & \int_{T}\left(\int_{\Lambda} \sum_{\nu, \mu \in \mathbb{Z}_{+}^{n}}\left|\left\langle\widehat{F}_{t}(\lambda) \Phi_{\nu, \lambda}, \Phi_{\mu, \lambda}\right\rangle_{\mathscr{H}_{\lambda}}\right|^{2} \rho(\lambda) d \lambda\right) \\
& \operatorname{Det}(t)^{-((m+n) / m)} d m_{l}(t)<\infty .
\end{aligned}
$$

Therefore $\operatorname{Det}(t)^{-((m+n) / 2 m)}\left\langle\widehat{F}_{t}(\lambda) \Phi_{\nu, \lambda}, \Phi_{\mu, \lambda}\right\rangle_{\mathscr{H}_{\lambda}} \in L^{2}\left(T, d m_{l}(t)\right)$ as the function of the variable $t$ for $\nu, \mu \in \mathbb{Z}_{+}^{n}, \lambda \in \Lambda$ almost everywhere. Suppose $\lambda=t_{\lambda}^{*} \lambda_{\epsilon} \in \Lambda_{\epsilon}, t_{\lambda} \in T$. Set

$$
\psi_{\delta}^{\lambda}(t)=\psi_{\delta}\left(t_{\lambda} t\right)
$$

Obviously $\left\{\psi_{\delta}^{\lambda}: \delta \in \Delta\right\}$ is also an orthonormal basis of $L^{2}\left(T, d m_{l}(t)\right)$. Therefore we have

$$
\operatorname{Det}(t)^{-((m+n) / 2 m)}\left\langle\widehat{F}_{t}(\lambda) \Phi_{\nu, \lambda}, \Phi_{\mu, \lambda}\right\rangle_{\mathscr{H}_{\lambda}}=\sum_{\delta \in \Delta} b_{\delta}(\lambda, \nu, \mu) \psi_{\delta}^{\lambda}(t),
$$


i.e.,

$$
\operatorname{Det}(t)^{-((m+n) / 2 m)} \widehat{F}_{t}(\lambda)=\sum_{\nu, \mu \in \mathbb{Z}_{+}^{n}, \delta \in \Delta} b_{\delta}(\lambda, \nu, \mu) \psi_{\delta}^{\lambda}(t) P_{\nu, \mu} .
$$

We define the functions $f_{\nu, \delta}^{\epsilon}$ in terms of the Fourier transform by

$$
\widehat{f}_{\nu, \delta}^{\epsilon}(\lambda)= \begin{cases}\sum_{\mu \in \mathbb{Z}_{+}^{n}} b_{\delta}(\lambda, \nu, \mu) P_{0, \mu}, & \text { if } \lambda \in \Lambda_{\epsilon}, \\ 0, & \text { if } \lambda \notin \Lambda_{\epsilon} .\end{cases}
$$

It is clear that $f_{\nu, \delta}^{\epsilon} \in H_{0}^{\epsilon}, \epsilon \in E$. And we have

$$
\begin{aligned}
& \int_{N} \sum_{\epsilon \in E, \nu \in \mathbb{Z}_{+}^{n}, \delta \in \Delta}\left(W_{\phi_{0, \nu, \delta}^{\epsilon}} f_{\nu, \delta}^{\epsilon}(x, \zeta, t)\right) \pi^{\lambda}(x, \zeta) d x d \zeta \\
& =\operatorname{Det}(t)^{((m+n) / 2 m)} \sum_{\epsilon \in E, \nu \in \mathbb{Z}_{+}^{n}, \delta \in \Delta} \widehat{f}_{\nu, \delta}^{\epsilon}(\lambda) \widehat{\phi}_{0, \nu, \delta}^{\epsilon}\left(t^{*} \lambda\right)^{*} \\
& =\operatorname{Det}(t)^{((m+n) / 2 m)} \sum_{\epsilon \in E, \nu \in \mathbb{Z}_{+}^{n}, \delta \in \Delta} b_{\delta}(\lambda, \nu, \mu) \psi_{\delta}^{\lambda}(t) P_{\nu, \mu} \\
& =\widehat{F}_{t}(\lambda) .
\end{aligned}
$$

Therefore we obtain

$$
F(x, \zeta, t)=\sum_{\epsilon \in E, \nu \in \mathbb{Z}_{+}^{n}, \delta \in \Delta} W_{\phi_{0, \nu, \delta}^{\epsilon}} f_{\nu, \delta}^{\epsilon}(x, \zeta, t)
$$

The proof of Theorem 5.1 is completed.

Let $M$ be the characteristic function of $\Omega$ defined by

$$
M(x)=\int_{\Omega^{*}} \mathrm{e}^{-2 \pi\langle\lambda, x\rangle} d \lambda .
$$

Similarly, the characteristic function $M^{*}$ of $\Omega^{*}$ is defined by

$$
M^{*}(\lambda)=\int_{\Omega} \mathrm{e}^{-2 \pi\langle\lambda, x\rangle} d x
$$

Then we have

$$
\begin{aligned}
M(g x) & =\operatorname{Det}(g)^{-1} M(x), \\
M^{*}\left(g^{*} \lambda\right) & =\operatorname{Det}(g)^{-1} M^{*}(\lambda),
\end{aligned}
$$


for $g \in \operatorname{Aut}(\Omega)$. Suppose $y=t e, t \in T$. Because $M(x) d x$ is an $\operatorname{Aut}(\Omega)$ invariant measure, there exists a constant $C$ such that $d m_{l}(t)=C M(y) d y$. Without loss of generality, we may assume that $C=M(e)^{(m+n) / m}$.

We have identified $P$ with $D$ according to the bijection

$$
(x, \zeta, t) \mapsto(x+i(y+\Phi(\zeta, \zeta)), \zeta),
$$

where $y=t e$. Then we have

$$
\operatorname{Det}(t)^{-((m+n) / m)} d x d \zeta d m_{l}(t)=M(y)^{((2 m+n) / m)} d x d y d \zeta,
$$

which turns out to be the $G_{a}(D)$-invariant measure on $D$. We can regard $L^{2}(P)$ as $L^{2}\left(D, M(y)^{((2 m+n) / m)} d x d y d \zeta\right)$, the space of square integrable functions on $D$ with respect to $G_{a}(D)$-invariant measure $M(y)^{((2 m+n) / m)}$ $d x d y d \zeta$. Then Theorem 5.1 gives the direct sum decomposition of $L^{2}(D, M$ $\left.(y)^{((2 m+n) / m)} d x d y d \zeta\right)$.

Suppose $(z, \zeta) \in D . \quad$ Set $x=\operatorname{Re} z, y=\operatorname{Im} z-\Phi(\zeta, \zeta)$. Then $d z d \zeta=$ $d x d y d \zeta$. Let

$$
L^{2}(D)=\left\{F(z, \zeta):\|F\|_{L^{2}(D)}=\left(\int_{D}|F(z, \zeta)|^{2} d z d \zeta\right)^{(1 / 2)}<\infty\right\} .
$$

The Bergman space $A(D)$ is the subspace of all holomorphic functions in $L^{2}(D)$.

Let $\phi \in A W_{0}^{\epsilon}, f \in H_{0}^{\epsilon}$. We define the revised wavelet transform $\widetilde{W}_{\phi}$ of $f$ with respect to $\phi$ by

$$
\begin{aligned}
\widetilde{W}_{\phi} f(x+i(t e+\Phi(\zeta, \zeta)), \zeta)= & C_{\phi}^{-(1 / 2)} M(e)^{((2 m+n) / 2 m)} \operatorname{Det}(t)^{-((2 m+n) / 2 m)} \\
& W_{\phi} f(x, \zeta, t) .
\end{aligned}
$$

Set

$$
\widetilde{A}_{\nu, \delta}^{\epsilon}=\left\{\widetilde{W}_{\phi_{0, \nu, \delta}^{\epsilon}} f: f \in H_{0}^{\epsilon}\right\}
$$

The direct consequence of Theorem 5.1 is

\section{Theorem 5.2.}

$$
L^{2}(D)=\bigoplus_{\epsilon \in E, \nu \in \mathbb{Z}_{+}^{n}, \delta \in \Delta} \widetilde{A}_{\nu, \delta}^{\epsilon}
$$


It is easy to see that the reproducing kernel of $\widetilde{A}_{\nu, \delta}^{\epsilon}$ is given by

$$
\begin{aligned}
& K_{\nu, \delta}^{\epsilon}\left((x+i(t e+\Phi(\zeta, \zeta)), \zeta),\left(x_{1}+i\left(t_{1} e+\Phi\left(\zeta_{1}, \zeta_{1}\right)\right), \zeta_{1}\right)\right) \\
&= M(\mathrm{e})^{((2 m+n) / m)} \operatorname{Det}\left(t t_{1}\right)^{-((2 m+n) / 2 m)} \\
& \int_{N} \pi\left(x_{1}, \zeta_{1}, t_{1}\right) \phi_{0, \nu, \delta}^{\epsilon}(a, \alpha) \overline{\pi(x, \zeta, t) \phi_{0, \nu, \delta}^{\epsilon}(a, \alpha)} d a d \alpha \\
&= M(\mathrm{e})^{((2 m+n) / m)} \operatorname{Det}\left(t t_{1}\right)^{-(1 / 2)} \int_{\Lambda_{\epsilon}} \operatorname{tr}\left(\widehat{\phi}_{0, \nu, \delta}^{\epsilon}\left(t^{*} \lambda\right)^{*}\right. \\
&\left.\pi^{\lambda}(x, \zeta)^{*} \pi^{\lambda}\left(x_{1}, \zeta_{1}\right) \widehat{\phi}_{0, \nu, \delta}^{\epsilon}\left(t_{1}^{*} \lambda\right)\right) \rho(\lambda) d \lambda .
\end{aligned}
$$

We select the orthonormal basis $\left\{\psi_{\delta}: \delta \in \Delta\right\}$ of $L^{2}\left(T, d m_{l}(t)\right)$ such that $\psi_{0} \in\left\{\psi_{\delta}: \delta \in \Delta\right\}$, where $\psi_{0}$ is defined by

$$
\psi_{0}(t)=M(\mathrm{e})^{-((2 m+n) / 2 m)} \mathrm{e}^{-2 \pi\left\langle\lambda_{0}, t e\right\rangle} M^{*}\left(2 t^{*} \lambda_{0}\right)^{-(1 / 2)},
$$

then

$$
\widehat{\phi}_{0,0,0}^{0}(\lambda)= \begin{cases}M(\mathrm{e})^{-((2 m+n) / 2 m)} \mathrm{e}^{-2 \pi\langle\lambda, e\rangle} M^{*}(2 \lambda)^{-(1 / 2)} P_{0,0}, & \text { if } \lambda \in \Omega^{*}, \\ 0, & \text { if } \lambda \notin \Omega^{*}\end{cases}
$$

The reproducing kernel of $\widetilde{A}_{0,0}^{0}$ is given by

$$
\begin{aligned}
& K_{0,0}^{0}\left((x+i(t e+\Phi(\zeta, \zeta)), \zeta),\left(x_{1}+i\left(t_{1} e+\Phi\left(\zeta_{1}, \zeta_{1}\right)\right), \zeta_{1}\right)\right) \\
&= M(\mathrm{e})^{((2 m+n) / m)} \operatorname{Det}\left(t t_{1}\right)^{-(1 / 2)} \int_{\Omega^{*}} \operatorname{tr}\left(\widehat{\phi}_{0,0,0}^{0}\left(t^{*} \lambda\right)^{*}\right. \\
&\left.\pi^{\lambda}(x, \zeta)^{*} \pi^{\lambda}\left(x_{1}, \zeta_{1}\right) \widehat{\phi}_{0,0,0}^{0}\left(t_{1}^{*} \lambda\right)\right) \rho(\lambda) d \lambda \\
&= \int_{\Omega^{*}} \mathrm{e}^{-2 \pi\left\langle\lambda, t e+t_{1} e\right\rangle} M^{*}(2 \lambda)^{-1} \\
&\left\langle\pi^{\lambda}\left(x_{1}, \zeta_{1}\right) \Phi_{0, \lambda}, \pi^{\lambda}(x, \zeta) \Phi_{0, \lambda}\right\rangle \mathscr{H}_{\lambda} \rho(\lambda) d \lambda .
\end{aligned}
$$

It is easy to compute that

$$
\left\langle\pi^{\lambda}\left(x_{1}, \zeta_{1}\right) \Phi_{0, \lambda}, \pi^{\lambda}(x, \zeta) \Phi_{0, \lambda}\right\rangle \mathscr{H}_{\lambda}=\mathrm{e}^{-2 \pi\left\langle\lambda, i\left(x_{1}-x\right)+\Phi\left(\zeta_{1}, \zeta_{1}\right)+\Phi(\zeta, \zeta)-2 \Phi\left(\zeta, \zeta_{1}\right)\right\rangle} .
$$

Therefore

$$
K_{0,0}^{0}\left((z, \zeta),\left(z_{1}, \zeta_{1}\right)\right)=\int_{\Omega^{*}} \mathrm{e}^{-2 \pi\left\langle\lambda, i\left(\overline{z_{1}}-z\right)-2 \Phi\left(\zeta, \zeta_{1}\right)\right\rangle} M^{*}(2 \lambda)^{-1} \rho(\lambda) d \lambda,
$$

which is exactly the Bergman kernel [19]. So $A_{0,0}^{0}$ is nothing but the Bergman space $A(D(\Phi, \Omega))$. 


\section{Radon transform on $N$}

The Radon transform on the Heisenberg group $H_{n}$ was defined by Strichartz [29]. In the same way, we define the Radon transform $R$ on $N$ by

$$
\begin{aligned}
R(f)(x, \zeta) & =\int_{V} f((x, \zeta)(0, w)) d w \\
& =\int_{V} f(x+2 \operatorname{Im} \Phi(\zeta, w), \zeta+w) d w
\end{aligned}
$$

We introduce the partial Fourier transforms $\mathscr{F}_{\lambda}$ and $\mathscr{F}$. For $\lambda$ in $\Lambda, \mathscr{F}_{\lambda}$ is used to represent the Fourier transform on $\mathscr{H}_{\lambda}$ defined by

$$
\mathscr{F}_{\lambda}(\phi)(\xi)=\int_{E_{\lambda}} \phi(\sigma) \exp (-2 \pi i \sigma \cdot \xi) \vartheta_{\lambda}(d \sigma), \quad \phi \in \mathscr{H}_{\lambda} .
$$

And $\mathscr{F}(f)$ is the Fourier transform of $f$ with respect to $x \in U$ alone, i.e.,

$$
\mathscr{F}(f)(\lambda, \zeta)=\int_{U} f(x, \zeta) \exp (-2 \pi i\langle\lambda, x\rangle) d x
$$

By (3.9) we have

$$
\begin{aligned}
\left(\widehat{R(f)}(\lambda) \Phi_{\nu, \lambda}\right)(\tau)= & \int_{U \times V} R(f)(x, \zeta) \times \exp (-2 \pi i\langle\lambda, x\rangle) \\
& \exp (\pi i \xi \cdot \eta) \exp (-2 \pi i \eta \cdot \tau) \Phi_{\nu, \lambda}(\tau-\xi) d x d \zeta
\end{aligned}
$$

where $\zeta=\xi+J_{\lambda} \eta, \xi, \eta \in E_{\lambda}$. Let $w=\mu+J_{\lambda} \gamma, \mu, \gamma \in E_{\lambda}$. It follows from (3.2) and (3.3) that

$$
\begin{aligned}
2 \pi i\langle\lambda, 2 \operatorname{Im} \Phi(\zeta, w)\rangle & =\pi i \operatorname{Im} H_{\lambda}(\zeta, w) \\
& =\pi i B_{\lambda}(\zeta, w) \\
& =-\pi i(\gamma \cdot \xi-\eta \cdot \mu) .
\end{aligned}
$$

Notice that the recursion formula for Hermite polynomials [28]

$$
\Phi_{\nu, \lambda}(-\eta)=(-1)^{|\nu|} \Phi_{\nu, \lambda}(\eta)
$$


where $|\nu|=\sum_{j}^{n} \nu_{j}$, by (3.6), we get

$$
\begin{aligned}
\left(\widehat{R(f)}(\lambda) \Phi_{\nu, \lambda}\right)(\tau)= & \int_{U V}\left(\int_{V} f(x+2 \operatorname{Im} \Phi(\zeta, w), \zeta+w) d w\right) \\
& \exp (-2 \pi i\langle\lambda, x\rangle) \exp (\pi i \xi \cdot \eta) \\
& \exp (-2 \pi i \eta \cdot \tau) \Phi_{\nu, \lambda}(\tau-\xi) d x d \zeta \\
= & 4^{-n}\left|\operatorname{Det} M_{\lambda}(\beta)\right|^{-1} \int_{V} \mathscr{F}(f)(\lambda, w) \\
& \left(\int_{E_{\lambda} E_{\lambda}} \exp (-\pi i(\gamma \cdot \xi-\eta \cdot \mu)) \exp (\pi i \xi \cdot \eta)\right. \\
& \left.\exp (-2 \pi i \eta \cdot \tau) \Phi_{\nu, \lambda}(\tau-\xi) \vartheta_{\lambda}(d \xi) \vartheta_{\lambda}(d \eta)\right) d w \\
= & 4^{-n}\left|\operatorname{Det} M_{\lambda}(\beta)\right|^{-1} \int_{V} \mathscr{F}(f)(\lambda, w) \exp (\pi i \mu \cdot \gamma) \\
& \exp (-2 \pi i \gamma \cdot \tau)\left(\int_{E_{\lambda}} \mathscr{F} \lambda\left(\Phi_{\nu, \lambda}\right)\left(\frac{\eta-\gamma}{2}\right)\right. \\
& \left.\exp (\pi i(\mu-\tau)(\eta-\gamma)) \vartheta_{\lambda}(d \eta)\right) d w \\
= & 2^{-n}\left|\operatorname{Det} M_{\lambda}(\beta)\right|^{-1} \int_{V} \mathscr{F}(f)(\lambda, w) \exp (\pi i \mu \cdot \gamma) \\
& \quad \exp (-2 \pi i \gamma \cdot \tau) \Phi_{\nu, \lambda}(\mu-\tau) d w \\
= & (-1)^{|\nu|} 2^{-n}\left|\operatorname{Det} M_{\lambda}(\beta)\right|^{-1}\left(\widehat{f}(\lambda) \Phi_{\nu, \lambda}\right)(\tau) .
\end{aligned}
$$

Thus we have

Theorem 6.1. Let $f \in L^{2}(N)$. Then

$$
\widehat{R(f)}(\lambda)=2^{-n}\left|\operatorname{Det} M_{\lambda}(\beta)^{-1}\right| \widehat{f}(\lambda) S
$$

where $S=\sum_{\nu \in \mathbb{Z}_{+}^{n}}(-1)^{|\nu|} P_{\nu}$ is a unitary operator on $\mathscr{H}_{\lambda}$.

It is known that Det $M_{\lambda}(\beta)$ is a real homogeneous polynomial of degree $n$ on $U^{\prime} \cong R^{m}\left[24\right.$, p. 35]. We put $P(\lambda)=\operatorname{Det} M_{\lambda}(\beta)$.

Let $x=\left(x_{1}, x_{2}, \ldots, x_{m}\right), f \in \mathscr{S}(N)$, it is obvious that

$$
\int_{U} \frac{\partial f}{\partial x_{j}}(x, \zeta) \exp (-2 \pi i\langle\lambda, x\rangle) d x=\left(2 \pi i \lambda_{j}\right) \mathscr{F}(f)(\lambda, \zeta) .
$$


Write $D=\left(\frac{1}{i} \frac{\partial}{\partial x_{1}}, \frac{1}{i} \frac{\partial}{\partial x_{2}}, \ldots, \frac{1}{i} \frac{\partial}{\partial x_{m}}\right), L=P(D)$. Such type operators has been considered by Gindikin [9]. It is not difficult to see that the partial differential operator $L$ satisfies

$$
\int_{U} L(f)(x, \zeta) \exp (-2 \pi i\langle\lambda, x\rangle) d x=(2 \pi)^{n} \operatorname{Det} M_{\lambda}(\beta) \mathscr{F}(f)(\lambda, \zeta) .
$$

Therefore we have

$$
\widehat{L(f)}(\lambda)=(2 \pi)^{n} \operatorname{Det} M_{\lambda}(\beta) \widehat{f}(\lambda)
$$

It follows that

$$
\widehat{L R(f)}(\lambda)=\widehat{R L(f)}(\lambda)=\pi^{n} \operatorname{sgn}\left(\operatorname{Det} M_{\lambda}(\beta)\right) \widehat{f}(\lambda) S,
$$

where $\operatorname{sgn}(\cdot)$ denotes the symbol function. Then we obtain the following inversion formula of the Radon transform on $N$.

$$
R^{-1}=\pi^{-2 n} L R L
$$

This is an extension of that on the Heisenberg group [29].

For a function $f \in L^{2}(N), R(f)$ may not belong to $L^{2}(N)$ [29]. We should find a dense subspace of $L^{2}(N)$, on which the formula (6.10) holds.

Theorem 6.2. Let

$$
\begin{aligned}
\mathscr{S}_{R}(N)= & \left\{f \in \mathscr{S}(N): \int_{\Lambda}\|\widehat{f}(\lambda)\|_{\mathrm{HS}}^{2}\left|\operatorname{Det} M_{\lambda}(\beta)\right|^{(2 j+1)} d \lambda<\right. \\
& +\infty, \text { for all } j \in \mathbb{Z}\} .
\end{aligned}
$$

Then $\mathscr{S}_{R}(N)$ is a dense subspace of $L^{2}(N)$ and

$$
R^{-1}(f)=\pi^{-2 n} L R L(f)
$$

for all $f \in \mathscr{S}_{R}(N)$.

Proof. Consider the subspace $L_{R}^{2}(N)$ of $L^{2}(N)$ defined by

$$
\begin{aligned}
L_{R}^{2}(N)= & \left\{f \in L^{2}(N): \int_{\Lambda}\|\widehat{f}(\lambda)\|_{\mathrm{HS}}^{2}\left|\operatorname{Det} M_{\lambda}(\beta)\right|^{(2 j+1)} d \lambda<\right. \\
& +\infty, \text { for all } j \in \mathbb{Z}\} .
\end{aligned}
$$


It is clear that $L_{R}^{2}(N)$ is a dense subspace of $L^{2}(N)$. By Theorem 6.1 and the Plancherel formula (3.10), we have

$$
\left\|R^{j}(f)\right\|_{L^{2}(N)}^{2}=4^{(-j+1) n} \int_{\Lambda}\|\widehat{f}(\lambda)\|_{\mathrm{HS}}^{2}\left|\operatorname{Det} M_{\lambda}(\beta)\right|^{(-2 j+1)} d \lambda .
$$

Thus $R$ is a bijection from $L_{R}^{2}(N)$ onto itself. Because $\mathscr{S}_{R}(N)=L_{R}^{2}(N) \bigcap$ $\mathscr{S}(N), L R L$ is well defined on $\mathscr{S}_{R}(N)$. Theorem 6.2 is proved.

\section{An inversion formula by using wavelets}

In this section, we establish an inversion formula of the Radon transform by use of the continuous wavelet, transform on $N$. By choosing suitable wavelets, the inversion formula of the Radon transform holds in the weak sense without the assumption of differentiability for $f$.

For simplicity, write $g_{t}(x, \zeta)=g\left(t^{-1} x, B(t)^{-1} \zeta\right)$ which deviates slightly from (3.15), we have

$$
\widehat{g_{t}}(\lambda)=\operatorname{Det}(t)^{((n+m) / m)} \widehat{g}\left(t^{*} \lambda\right) .
$$

We define the operator $\widetilde{W}_{g}$ by

$$
\widetilde{W}_{g} f=f * \widetilde{g}
$$

Then

$$
\left(\widehat{W_{g} f}\right)(\lambda)=\widehat{f}(\lambda) \widehat{g}(\lambda)^{*}
$$

Note that

$$
\operatorname{Det} M_{t^{*} \lambda}(\beta)=|\operatorname{Det}(B(t))|^{2} \operatorname{Det} M_{\lambda}(\beta)=\operatorname{Det}(t)^{n / m} \operatorname{Det} M_{\lambda}(\beta) \text {. }
$$

Applying (7.1), (7.2) together with (6.8), we have

$$
\begin{aligned}
\left(\widehat{\left.\widetilde{W}_{L(g)_{t}} f\right)(\lambda)}\right. & =\widehat{f}(\lambda) \widehat{L(g)_{t}}(\lambda)^{*} \\
& =\operatorname{Det}(t)^{((n+m) / m)} \widehat{f}(\lambda) \widehat{L(g)}\left(t^{*} \lambda\right)^{*} \\
& =(2 \pi)^{n} \operatorname{Det}(t)^{((2 n+m) / m)} \operatorname{Det} M_{\lambda}(\beta) \widehat{f}(\lambda) \widehat{g}\left(t^{*} \lambda\right)^{*}
\end{aligned}
$$


On the other hand, by (7.2), (6.8) and (7.1) we get

$$
\begin{aligned}
\left(\widehat{W_{g_{t}} L(f)}\right)(\lambda) & =\widehat{L(f)}(\lambda) \widehat{g}_{t}(\lambda)^{*} \\
& =(2 \pi)^{n} \operatorname{Det} M_{\lambda}(\beta) \operatorname{Det}(t)^{((n+m) / m)} \widehat{f}(\lambda) \widehat{g}\left(t^{*} \lambda\right)^{*} .
\end{aligned}
$$

Consequently,

$$
\left(\widetilde{W}_{L(g)_{t}} f\right)(x, \zeta)=\operatorname{Det}(t)^{n / m}\left(\widetilde{W}_{g_{t}} L(f)\right)(x, \zeta) .
$$

Theorem 7.1. Let $g \in \mathscr{S}_{R}(N) \cap A W_{\nu}^{\epsilon}, f \in \mathscr{S}_{R}(N) \cap H_{\nu}^{\epsilon}$. Then

$$
\left(\widetilde{W}_{L R L(g) t} R(f)\right)(x, \zeta)=\pi^{2 n} \operatorname{Det}(t)^{((m+3 n) / 2 m)}\left(W_{g} f\right)(x, \zeta, t) .
$$

Proof. By (7.6), we have

$$
\left(\widetilde{W}_{L R L(g)_{t}} R(f)\right)(x, \zeta)=\operatorname{Det}(t)^{n / m}\left(\widetilde{W}_{R L(g)_{t}} L R(f)\right)(x, \zeta) .
$$

Note that $\operatorname{sgn}\left(\operatorname{Det} M_{t^{*} \lambda}(\beta)\right)=\operatorname{sgn}\left(\operatorname{Det} M_{\lambda}(\beta)\right)$. By taking the Fourier transform on both sides of (7.8), it follows that

$$
\begin{aligned}
\left(\widetilde{W}_{L R L(g)_{t}} R(f)\right)(\lambda) & =\operatorname{Det}(t)^{n / m}\left(\widetilde{W}_{R L(g)_{t}} L R(\widehat{f})\right)(\lambda) \\
& =\operatorname{Det}(t)^{n / m} \widehat{L R(f)}(\lambda) \widehat{R L(g)} t(\lambda)^{*} \\
& =\operatorname{Det}(t)^{((m+2 n) / m)} \widehat{L R(f)}(\lambda) \widehat{R L(g)}\left(t^{*} \lambda\right)^{*} \\
& =\pi^{2 n} \operatorname{Det}(t)^{((m+2 n) / m)} \widehat{f}(\lambda) \widehat{g}\left(t^{*} \lambda\right)^{*} .
\end{aligned}
$$

But by the wavelet transform

$$
\widehat{W_{g}} f(\lambda)=\operatorname{Det}(t)^{((m+n) / 2 m)} \widehat{f}(\lambda) \widehat{g}\left(t^{*} \lambda\right)^{*},
$$

this completes the proof of (7.7).

In Theorem 7.1, we make the assumption of differentiability for $f$, which is needed in (7.8). This assumption can be removed. In fact, we can deduce (7.7) from the following computation. According to (6.10) and (7.3), we have

$$
\begin{aligned}
\left(\widetilde{W}_{L R L(g)_{t}} R(f)\right)(\lambda) & =\widehat{R(f)}(\lambda) \widehat{L R L(g)})_{t}(\lambda)^{*} \\
& \left.=\operatorname{Det}(t)^{((m+n) / m)} \widehat{R(f)}(\lambda) \widehat{L R L(g}\right)\left(t^{*} \lambda\right)^{*} \\
& =\pi^{2 n} \operatorname{Det}(t)^{((m+2 n) / m)} \widehat{f}(\lambda) \widehat{g}\left(t^{*} \lambda\right)^{*}
\end{aligned}
$$

We therefore have the following inverse Radon transform in the weak sense. 
Theorem 7.2. Let $\phi_{\nu}^{\epsilon} \in \mathscr{S}_{R}(N) \cap A W_{\nu}^{\epsilon}, f \in L_{R}^{2}(N) \cap H_{\nu}^{\epsilon}$. Then

$$
\begin{array}{r}
f(x, \zeta)=\frac{1}{\pi^{2 n} C_{\phi_{\nu}^{\epsilon}}} \int_{N \times T} \widetilde{W}_{L R L\left(\phi_{\nu}^{\epsilon}\right)_{t}} R(f)(a, \alpha) \\
U(a, \alpha, t) \phi_{\nu}^{\epsilon}(x, \zeta) \frac{d a d \alpha d m_{l}(t)}{\operatorname{Det}(t)^{((3 m+5 n) / 2 m)}}
\end{array}
$$

and

$$
\begin{aligned}
R^{-1}(f)(x, \zeta)= & \frac{1}{\pi^{2 n} C_{\phi_{\nu}^{\epsilon}}} \int_{N \times T} \widetilde{W}_{L R L\left(\phi_{\nu}^{\epsilon}\right)_{t}} f(a, \alpha) \\
& U(a, \alpha, t) \phi_{\nu}^{\epsilon}(x, \zeta) \frac{d a d \alpha d m_{l}(t)}{\operatorname{Det}(t)^{((3 m+5 n) / 2 m)}} .
\end{aligned}
$$

in the weak sense. Specifically, if $f \in \mathscr{S}_{R}(N) \cap H_{\nu}^{\epsilon}$, then the formula (7.10), (7.11) hold for all $(x, \zeta) \in N$.

It is easy to construct some wavelets which satisfy the condition in Theorem 7.2. Let $\psi \in C_{0}^{\infty}(T)$ satisfying $\operatorname{Det}(t) \geq \eta>0$ for $t \in \operatorname{supp} \psi \cdot \phi_{\nu, \mu, \delta}^{\epsilon}$ is defined by

$$
\widehat{\phi}_{\nu, \mu, \delta}^{\epsilon}(\lambda)= \begin{cases}\psi(t) P_{\nu, \mu}, & \text { if } \lambda=t^{*} \lambda_{\epsilon} \in \Lambda_{\epsilon}, \\ 0, & \text { if } \lambda \notin \Lambda_{\epsilon},\end{cases}
$$

then we have $\phi_{\nu, \mu, \delta}^{\epsilon} \in \mathscr{S}_{R}(N) \cap A W_{\nu}^{\epsilon}$.

If $f \in \mathscr{S}_{R}(N)$ (or $f \in L_{R}^{2}(N)$ ), then $f$ can be decomposed as $f=\sum_{\epsilon, \nu} f_{\nu}^{\epsilon}$ where $f_{\nu}^{\epsilon} \in \mathscr{S}_{R}(N) \cap H_{\nu}^{\epsilon}\left(\right.$ or $\left.f_{\nu}^{\epsilon} \in L_{R}^{2}(N) \cap H_{\nu}^{\epsilon}\right)$. Take $\phi_{\nu}^{\epsilon} \in \mathscr{S}_{R}(N) \cap A W_{\nu}^{\epsilon}$. It follows from (7.11) that

$$
\begin{array}{r}
R^{-1}(f)(x, \zeta)=\sum_{\epsilon, \nu} \frac{1}{\pi^{2 n} C_{\phi_{\nu}^{\epsilon}}} \int_{N \times T} \widetilde{W}_{L R L\left(\phi_{\nu}^{\epsilon}\right)_{t}} f_{\nu}^{\epsilon}(a, \alpha) \\
U(a, \alpha, t) \phi_{\nu}^{\epsilon}(x, \zeta) \frac{d a d \alpha d m_{l}(t)}{\operatorname{Det}(t)^{((3 m+5 n) / 2 m)}}
\end{array}
$$

holds for all $(x, \zeta) \in N$ (or in the weak sense).

\section{The symmetric case}

Of course, the most interested case is the symmetric Siegel domains of type II, which (together with the symmetric tube domains) are the unbounded realizations of the bounded symmetric domains. In this case, our results can 
be expressed in a more explicit form in terms of Jordan algebra. We shall only state the results without proof and refer the reader to [20] for some detailed calculation about the symmetric cones. A good reference on the Jordan algebras and the symmetric cones is the book [7] by J. Faraut and Korányi.

Let $U$ be a simple Euclidean Jordan algebra with the identity e. $\Omega$ is the associated symmetric cone. Then $D=D(\Omega, \Phi)$ determined by the symmetric cone $\Omega$ and an $\Omega$-positive Hermitian map $\Phi$ is a symmetric Siegel domain of type II. Suppose that $U$ has the dimension $m$, the rank $r$ and the degree $d . x \circ y$ denotes the Jordan product of $x$ and $y \cdot \operatorname{tr}(x)$ and $\operatorname{det}(x)$ are defined as in [7]. We also write $\Delta(x)$ instead of $\operatorname{det}(x)$. The inner product on $U$ is given by $\langle x, y\rangle=\operatorname{tr}(x \circ y)$. We select $K$ to be the isotropy subgroup of $G_{a}(D)$ at the point $(i e, 0)$. Because two maximal $\mathbb{R}$-triangular subgroups of a linear Lie group $G$ are conjugate with respect to an inner automorphism of $G$, there exist a Jordan frame $\left\{c_{1}, \ldots, c_{r}\right\}$ and the corresponding Peirce decomposition

$$
U=\bigoplus_{j \leq k} U_{j k}
$$

such that $T$ has the parameterization as

$$
T=\left\{t(u): u \in U_{+}\right\},
$$

where

$$
U_{+}=\left\{u=\sum_{j=1}^{r} u_{j} c_{j}+\sum_{j<k} u_{j k}: u_{j}>0, u_{j k} \in U_{j k}\right\} .
$$

The left Haar measure of $T$ is given by

$$
d m_{l}(t(u))=2^{r} \prod_{j=1}^{r} u_{j}^{-d(j-1)-1} d u .
$$

We identify $U^{\prime}$ with $U$ by the use of the inner product. Then

$$
\rho(\lambda)=4^{n} \Delta(\lambda)^{n / r} .
$$

Let

$$
E=\left\{\epsilon=\left(\epsilon_{1}, \epsilon_{2}, \ldots, \epsilon_{r}\right): \epsilon_{j}= \pm 1\right\}
$$

Set

$$
\lambda_{\epsilon}=\sum_{j=1}^{r} \epsilon_{j} c_{j} .
$$


All simple $T$-orbits of $U$ under the adjoint action are given by

$$
\Lambda_{\epsilon}=\left\{\lambda \in U: \lambda=t(u)^{*} \lambda_{\epsilon}, u \in U_{+}\right\}, \quad \epsilon \in E .
$$

Specifically, $\Lambda_{e}=\Lambda_{(1,1, \ldots, 1)}=\Omega$.

We can identify $\Omega$ with $T$ by identification of $x=t(u) e$ and $t(u)$. Then we have

$$
\Delta(x)^{-\frac{m}{r}} d x=d \mu_{l}(t(u))
$$

and

$$
\operatorname{Det}(t(u))=\Delta(x)^{(m / r)} .
$$

The characteristic function $M(x)$ of $\Omega$ is given by

$$
M(x)=\Gamma_{\Omega}\left(\frac{m}{r}\right)(2 \pi)^{-m} \Delta(x)^{-(m / r)},
$$

where $\Gamma_{\Omega}$ is the gamma function of the symmetric cone $\Omega$. Specifically,

$$
M(\mathrm{e})=\Gamma_{\Omega}\left(\frac{m}{r}\right)(2 \pi)^{-m}
$$

Let $\Delta_{j}(x), j=1, \ldots, r$, denote the principal minors. $\Delta_{j}^{*}(x)=\Delta_{j}(k x)$ where $k$ is an automorphism of $U$ such that

$$
k c_{j}=c_{r-j+1}, \quad j=1, \ldots, r .
$$

Let $\mathbf{s}=\left(s_{1}, \ldots, s_{r}\right)$. We set

$$
\Delta_{\mathbf{S}}^{*}(x)=\Delta_{1}^{*}(x)^{s_{1}-s_{2}} \cdots \Delta_{r-1}^{*}(x)^{s_{r-1}-s_{r}} \Delta_{r}^{*}(x)^{s_{r}} .
$$

For the transformation $\lambda=t(u)^{*} \lambda_{\epsilon}$, we have

$$
d m_{l}(t(u))=\left|\Delta_{\underline{\mathbf{s}}}^{*}(\lambda)\right|^{-1} d \lambda,
$$

where

$$
\underline{\mathbf{s}}=(1+d(r-1), 1+d(r-2), \ldots, 1) .
$$

Because

$$
\operatorname{Det} M_{\lambda}(\beta)=\Delta(\lambda)^{n / r},
$$


we have

$$
L=\Delta(D)^{n / r}
$$

Now all results in previous sections can be expressed in a more explicit form for the symmetric case. For example, Theorem 4.1 can be restated as follows.

Suppose $\phi(\neq 0)$ in $H_{\nu}^{\epsilon}$. Then $\phi \in A W_{\nu}^{\epsilon}$ if and only if

$$
C_{\phi}=\int_{\Lambda_{\epsilon}}\|\widehat{\phi}(\lambda)\|_{\mathrm{HS}}^{2}\left|\Delta_{\underline{\mathbf{s}}}^{*}(\lambda)\right|^{-1} d \lambda<\infty
$$

A concrete example of the symmetric Siegel domains of type II is the unbounded realization of the classical domain of type one. Let $V=M_{s, r}$ be the set of all $s \times r$ complex matrices, $U=H_{r}$ denotes all Hermitian matrices, and write $W=M_{r}=M_{r, r} . H_{r}$ is a simple Euclidean Jordan algebra with the Jordan product

$$
x \circ y=\frac{1}{2}(x y+y x) .
$$

$H_{r}$ has the dimension $m=r^{2}$, the rank $r$ and the degree $d=2$. The associated symmetric cone $\Omega$ consists of all complex Hermitian positive definite matrices in $H_{r}$. The trace function $\operatorname{tr}(x)$ and the determinant function $\operatorname{det}(x)$ are the usual ones. Set

$$
\begin{aligned}
\Phi: M_{s, r} \times M_{s, r} & \rightarrow M_{r}, \\
(\zeta, \eta) & \mapsto \Phi(\zeta, \eta)=\eta^{*} \zeta .
\end{aligned}
$$

$\Phi$ is an $\Omega$-positive Hermitian map, and

$$
D(\Omega, \Phi)=\left\{(z, \zeta) \in M_{r} \times M_{s, r}: \frac{z-z^{*}}{2 i}-\zeta^{*} \zeta>0\right\}
$$

is a symmetric Siegel domain of type II. This is the unbounded realization of the classical domain of type one. We select the Jordan frame $\left\{c_{1}, \ldots, c_{r}\right\}$ such that $c_{j}$ is a diagonal matrix with 1 in the $j$ th place and 0 in other positions. Then $T$ consists of all $r \times r$ upper triangular matrices with positive diagonal elements. The action of $T$ on $H_{r}$ is given by $t(x)=t x t^{*}$ and $\operatorname{Det}(t)=\operatorname{det}(t)^{2 r}$. All results for this case are expressed in a more explicit 
form $[12,13]$. For example, we have $L=(-i)^{s r}\left(\operatorname{det}\left(\frac{\partial}{\partial x_{j k}}\right)\right)^{s}$ and

$$
\begin{array}{r}
R^{-1}(f)(x, \zeta)=\frac{1}{\pi^{2 s} C_{\phi_{\nu}^{\epsilon}}} \int_{N \times T} \widetilde{W}_{L R L\left(\phi_{\nu}^{\epsilon}\right)_{t}} f(a, \alpha, t) \\
U(a, \alpha, t) \phi_{\nu}^{\epsilon}(x, \zeta) \frac{d a d \alpha d m_{l}(t)}{\operatorname{det}(t)^{3 r+5 s}} .
\end{array}
$$

\section{Acknowledgement}

The authors would like to thank Professor Detlef Müller for several valuable discussions on this subject of this paper. The first author was supported by the National Natural Science Foundation of China (No. 10371087, 10671041), the Science Research Foundation of Guangzhou Educational Bureau, the second author was supported by the National Natural Science Foundation of China (No. 10371004) and the Specialized Research Fund for the Doctoral Program of Higher Education of China (No. 20030001107).

\section{References}

[1] D. Bernier and K. F. Taylor, Wavelets from square-integrable representations, SIAM J. Math. Anal. 27 (1996), 594-608.

[2] C. K. Chui, An Introduction to Wavelets, Academic Press, Boston, MA, 1992.

[3] E. Damek, A. Hulanicki, D. Müller and M. M. Peloso, Pluriharmonic $H^{2}$ functions on symmetric irreducible Siegel domains, Geom. Funct Anal. 10 (2000), 1090-1117.

[4] I. Daubechies, Ten lectures on wavelets, CBMS-NSF, Regional Conf. Ser. in Math. 61, SIAM, Philidephia, PA, 1992.

[5] I. Daubechies, A. Grossmann and Y. Meyer, Painless nonorthogonal expansions, J. Math. Phys. 27 (1986), 1271-1283.

[6] M. Duflo and C. C. Moore, On the regular representation of a nonunimodular locally compact group, J. Funct. Anal. 21 (1976), 209-243.

[7] J. Faraut and A. Korányi, Analysis on Symmetric Cones, Oxford, 1994.

[8] H. G. Feichtinger and K. H. Gröchenig, Banach spaces related to integrable group representations and their atomic decomposition I, J. Funct. Anal. 86 (1989), 307-340. 
[9] S. G. Gindikin, Analysis on homogeneous domains, Uspekhi Math. Nauk. 19 (1964), 3-92; Russian Math. Survey 19 (4), 1-89.

[10] A. Grossmann, J. Morlet and T. Paul, Transforms associated to square integrable group representations I: General results, J. Math. Phys. 26 (1985), 2473-2479.

[11] J. X. He and H. P. Liu, Admissible wavelets associated with the affine automorphism group of the siegel upper half-plane, J. Math. Anal. Appl. 208 (1997), 58-70.

[12] J. X. He and H. P. Liu, Admissible wavelets associated with the classical domain of type one, Approx. Theory Appl. 14 (1998), 89-105.

[13] J. X. He and H. P. Liu, Inversion of the Radon transform associated with the classical domain of type one, Int. J. Math. 16 (2005), 875-887.

[14] C. E. Heil and D. F. Walnut, Continuous and discrete wavelet transforms, SIAM Rev. 31 (1989), 628-666

[15] S. Helgason, The Radon transform, Second Edition, Birkhäuser, Boston Basel, Berlin, 1999.

[16] M. Holschneider, Inverse Radon transforms through inverse wavelet transforms, Inverse Prob. 7 (1991), 853-861.

[17] Q. T. Jiang, Wavelet transform and orthogonal decomposition of $L^{2}$ space on the Cartan domain BDI $(q=2)$, Trans. Am. Math. Soc. 349 (1997), 2049-2068.

[18] S. Kaneyeki, Homogenous bounded domains and Siegel domains, Lecture Notes in Math., 241, Springer-Verlag, Berlin, New York, 1971.

[19] A. Korányi and E. M. Stein, $H^{2}$ spaces of general half-planes, Studia Math. 44 (1972), 379-388.

[20] H. P. Liu, Wavelet transforms and symmetric tube domains, J. Lie Theory, 8 (1998), 351-366.

[21] H. P. Liu and L. Z. Peng, Admissible wavelets associated with the Heisenberg group, Pacific J. Math. 180 (1997), 101-123.

[22] S. Murakami, On automorphisms of Siegel domains, Lecture Notes in Math., Vol. 286, Springer-Verlag, Berlin, New York, 1972.

[23] M. M. Nessibi and K. Trimèche, Inversion of the Radon transform on the Laguerre hypergroup by using generalized wavelets, J. Math. Anal. Appl. 208 (1997), 337-363. 
[24] R. D. Ogden and S. Vági, Harmonic analysis of a nilpotent group and function theory on Siegel domains of type II, Adv. Math. 33 (1979), $31-92$.

[25] I. I. Pjateckii-Šapiro, Geometry of classical domains and theory of automorphic functions, Fizmatgiz, Moscow, 1961 (Russian); English translation, Gordon and Breach, New York, 1969.

[26] B. Rubin, The Calderón formula, windowed X-ray transforms and Radon transforms in $L^{p}$-spaces, J. Fourier Anal. Appl. 4 (1998), 175197.

[27] B. Rubin, Fractional calculus and wavelet transforms in integral geometry, Fract. Calc. Appl. Anal. 1 (1998), 193-219.

[28] J. B. Seaborn, Hypergeometric functions and their applications, Springer-Verlag, New York, Berlin Heidelberg, London, 1991.

[29] R. S. Strichartz, $L^{p}$ harmonic analysis and Radon transforms on the Heisenberg group, J. Funct. Anal. 96 (1991), 350-406.

[30] S. Vági, Harmonic analysis on Carton and Siegel domains, Studies in Harmonic Analysis ed. J. A. Ash, MAA, 1976, pp. 257-309.

[31] R. A. Zuidwijk, Directional and time-scale wavelet analysis, SIAM J. Math. Anal. 31 (2000), 416-430.

Department of Mathematics

School of Mathematics and Information Sciences

GuANGZHOU UnIVERSITY

GuANGZHOU 510006

PR CHINA

E-mail address: h_jianxun@hotmail.com

LMam, School of Mathematical Sciences

PEKING University

BEIJING 100871

PR CHINA

E-mail address: hpliu@math.pku.edu.cn

ReCEIVEd January 11, 2006 